\title{
Imaging Three-Dimensional Microvascular Networks of Brain with Synchrotron Radiation Microangiography
}

\author{
Ming Song ${ }^{1,2,3}$
}

Received: 19 February 2020/Accepted: 13 March 2020/Published online: 2 April 2020

(C) Shanghai Institutes for Biological Sciences, CAS 2020

The adult human brain represents about $2 \%$ of the body weight, yet consumes about $20 \%$ of the body's total energy. A key constituent in meeting this high energy demand is a complex cerebral vascular system. A detailed knowledge of this system is important for understanding the basic principles of cerebral blood flow and its coupling to neural processing and neuropathological alterations in brain diseases. In this issue, $\mathrm{Gu}$ and collaborators utilized synchrotron radiation (SR) microangiography to acquire high-resolution (about $5 \mu \mathrm{m}$ ), high-contrast images of the brain tissue of rats under both normal and epileptic seizure conditions without the use of any contrast agent or labeling marker [1]. This study demonstrates that SR microangiography can provide systematic and detailed views of cerebrovascular anatomy at the micron level.

The diameters of cortical vessels range from several hundreds of microns (arteries/veins) to a few microns (capillaries). Although the detection of cortical vessels at the macro-level $(>500 \mu \mathrm{m})$ has been successful using current techniques such as digital subtraction angiography, magnetic resonance angiography, and computed tomography angiography, methods to detect microvascular networks $(<100 \mu \mathrm{m})$ are still scarce. In order to estimate the diameters of capillaries, submicron spatial resolution is

Ming Song

msong@nlpr.ia.ac.cn

1 National Laboratory of Pattern Recognition, Institute of Automation, Chinese Academy of Sciences, Beijing 100190, China

2 Brainnetome Center, Institute of Automation, Chinese Academy of Sciences, Beijing 100190, China

3 University of the Chinese Academy of Sciences, Beijing 100049, China required. Classical studies of microvascular networks are based on a combination of tissue staining (e.g. filling with Indian ink) or immunohistochemistry with microscopic imaging techniques. However, most of these studies did not make direct images of the whole brain in a threedimensional fashion, rather than isolated slices of brain tissue after tissue removal and manipulation with aggressive fixatives, various chemical detergents, and geometric distortions [2].

Recently, some new technologies have opened promising avenues for imaging the detailed three-dimensional vascular network topology of the brain, although these methods can only be applied to small animals at present. One of these novel technologies is based on microscopic optical imaging. For example, researchers have developed the all-optical histology technique [3], whereby two-photon microscopy is used to image fluorescently-labeled vasculature. Although this method has the ability to measure vascular diameters in vivo, the field of view is normally limited to a few hundred microns, without covering the entire brain of a mammal. With the advent of tissue clearing methods, selective plane illumination microscopy or ultramicroscopy is becoming an increasingly powerful approach to acquire and reconstruct these fluorescentlylabeled vessels [4-6]. Unfortunately, tissue clearing technology can only be used in specimens. Another possible approach is the use of SR microangiography, where a high photon flux is exploited to acquire detailed high-resolution images of microvessels even down to a diameter of $1 \mu \mathrm{m}$ or even higher resolution. In a specimen, SR microangiography can quickly characterize the three-dimensional morphology without sectioning. In particular, a recent study reported that the total volume of a mouse brain specimen can be acquired with micron resolution in about 15 min [7]. In fact, SR microangiography has the potential 
to generate images of the cerebral vascular networks in large animals in vivo [8], which is impossible with the methods based on tissue-clearing technology. In addition, one major advantage of SR microangiography is its ability to differentiate vessels without the use of any contrast agent or labeling marker. Therefore, when combined with tomography algorithms, SR microangiography can permit very detailed analysis of the whole brain vasculature.

In summary, the cerebrovascular system plays an important role in brain function, both in health and disease. Novel cerebrovascular imaging approaches are needed, especially for detecting micro-vascular anatomy and physiology at the micron level. The study of $\mathrm{Gu}$ and collaborators [1] suggests that SR microangiography could be one of the most promising approaches to addressing relevant questions regarding the involvement of microvascular network alterations in brain diseases.

Acknowledgements This editorial was supported by the National Natural Science Foundation of China (31870984).

\section{References}

1. Gu P, Xu ZH, Cao YZ, Liao SH, Deng QF, Yin XZ, et al. Synchrotron radiation-based three-dimensional visualization of angioarchitectural remodeling in hippocampus of epileptic rats. Neurosci Bull 2020, 36: 333-345. https://doi.org/10.1007/s12264019-00450-0.

2. Zhu X, Xia Y, Wang X, Si K, Gong W. Optical brain imaging: a powerful tool for neuroscience. Neurosci Bull 2017, 33: 95-102.

3. Tsai PS, Friedman B, Ifarraguerri AI, Thompson BD, Lev-Ram V, Schaffer CB, et al. All-optical histology using ultrashort laser pulses. Neuron 2003, 39: 27-41.

4. Ertuerk A, Becker K, Jaehrling N, Mauch CP, Hojer CD, Egen JG, et al. Three-dimensional imaging of solvent-cleared organs using 3DISCO. Nature Protocols 2012, 7: 1983-1995.

5. Susaki EA, Tainaka K, Perrin D, Yukinaga H, Kuno A, Ueda HR. Advanced CUBIC protocols for whole-brain and whole-body clearing and imaging. Nat Protocols 2015, 10: 1709-1727.

6. Rocha MD, Duering DN, Bethge P, Voigt FF, Hildebrand S, Helmchen $\mathrm{F}$, et al. Tissue clearing and light sheet microscopy: imaging the unsectioned adult zebra finch brain at cellular resolution. Front Neuroanat 2019, 13, 13.

7. Massimi L, Bukreeva I, Santamaria G, Fratini M, Corbelli A, Brun F, et al. Exploring Alzheimer's disease mouse brain through X-ray phase contrast tomography: From the cell to the organ. Neuroimage 2019, 184: 490-495.

8. Schueltke E, Kelly ME, Nemoz C, Fiedler S, Ogieglo L, Crawford $\mathrm{P}$, et al. Dual energy CT at the synchrotron: A piglet model for neurovascular research. Eur J Radiol 2011, 79: 323-327. 